\title{
Optimización del proceso industrial del poliuretano a través del diseño estadístico de Taguchi
}

\section{Optimization of the industrial process of polyurethane through the statistical design of Taguchi}

\author{
LAGUNAS-BERNABÉ, Ignacio†*, HUITRÓN-CID, María De Lourdes, ALVARADO-ORTIZ, Jesús \\ y PÉREZ-MIRELES, Emilio
}

División de Ingeniería Química, Tecnológico de Estudios Superiores de Jilotepec

División de Licenciatura en Administración, Tecnológico de Estudios Superiores de Jilotepec

ID $1^{\mathrm{er}}$ Autor: Ignacio, Lagunas-Bernabé / ORC ID: 0000-0001-6018-6079, Researcher ID Thomson: I-2780-2018, CVU CONACYT ID: 97810

ID $1^{\text {er }}$ Coautor: María De Lourdes, Huitrón-Cid /ORC ID: 0000-0002-5133-6175, Researcher ID Thomson: X-7088-2019, CVU CONACYT ID: 989548

\author{
ID $2^{\text {do }}$ Coautor: Jesús, Alvarado-Ortiz / ORC ID: 0000-0003-1655-5214, Researcher ID Thomson: X-7055-2019, CVU \\ CONACYT ID: 1000689 \\ ID $3^{\text {er }}$ Coautor: Emilio, Pérez-Mireles / ORC ID: 0000-0001-8790-6291, arXiv Author ID: 2650949, CVU CONACYT ID: \\ 562369
}

\section{Resumen}

En una empresa del ramo de polímeros, ubicado en Jilotepec, Estado de México, presenta la necesidad de resolver algunos problemas en la elaboración de espuma de poliuretano como son los códigos 25 STD BCQ y 64 $\mathrm{FB}$, las cuales deben cumplir con una densidad y compresión específicas. Se empleo el diseño experimental de Taguchi, que permite diseñar experimentos que usualmente requieren sólo una fracción de las combinaciones factoriales completas, cada factor puede ser evaluado de manera independiente, de manera que el efecto de un factor no ejerce influencia en la estimación de otro factor. Se evaluaron 7 factores con dos niveles cada uno: índice de TDI, agua, poliol, amina, catalizador Z-7, silicón y cloruro de metilo. Para el cálculo de la densidad ( $\mathrm{kg} / \mathrm{m} 3)$, se pesaron cada una de las muestras obtenidas en una báscula analítica y se obtuvo el volumen de cada una de ellas. La compresión se midió en una maquina universal Instron ${ }^{\circledR}$. La nueva formulación optimizada para la espuma 25 STD BCQ logró una densidad de 14-15 kg/m3 y una compresión 27-33lbf., mientras que la espuma 64 FB logró una densidad de $23-25 \mathrm{~kg} / \mathrm{m} 3$ y una compresión 58-68 lbf., estos resultados cumplen satisfactoriamente las especificaciones del cliente

Poliuretano, Taguchi, Densidad

\begin{abstract}
In a company of the polymer branch, located in Jilotepec, State of Mexico, presents the need to solve some problems in the manufacture of polyurethane foam such as codes 25 STD BCQ and $64 \mathrm{FB}$, which must comply with a density and compression specific. We used the experimental design of Taguchi, which allows to design experiments that usually require only a fraction of the complete factor combinations, each factor can be evaluated independently, so that the effect of one factor does not influence the estimation of another factor. Seven factors were evaluated with two levels each: index of TDI, water, polyol, amine, catalyst Z-7, silicon and methyl chloride. To calculate the density $(\mathrm{kg} / \mathrm{m} 3)$, each of the samples obtained was weighed on an analytical scale and the volume of each one was obtained. The compression was measured on an Instron ${ }^{\circledR}$ universal machine. The new formulation optimized for the foam 25 STD BCQ achieved a density of $14-15 \mathrm{~kg} / \mathrm{m} 3$ and a compression of 27-33 lbf., While the foam $64 \mathrm{FB}$ achieved a density of $23-25 \mathrm{~kg} / \mathrm{m} 3$ and a compression 58- $68 \mathrm{lbf}$., These results satisfactorily meet customer specifications.
\end{abstract}

Polyurethane, Taguchi, Density

Citación: LAGUNAS-BERNABÉ, Ignacio, HUITRÓN-CID, María De Lourdes, ALVARADO-ORTIZ, Jesús y PÉREZMIRELES, Emilio. Optimización del proceso industrial del poliuretano a través del diseño estadístico de Taguchi. Revista de Tecnologías en Procesos Industriales. 2019 3-8: 8-14

\footnotetext{
* Correspondencia del Autor (Correo electrónico: lagunas.ixo7@gmail.com)

$\dagger$ Investigador contribuyendo como primer autor.
} 


\section{() ECORFAN- Taiwan}

\section{Introducción}

Desde el descubrimiento de los poliuretanos realizado por Otto Bayer en 1937, estos polímeros han estado presentes en diversos sectores de la industria, desde los colchones y sillas inyectadas, fabricadas en espuma flexible, hasta la espuma rígida utilizada como aislantes térmicos debido a su baja conductividad o como elastómeros que contienen celdas cerradas y se utilizan principalmente en partes de vehículos como los volantes o para la fabricación de suelas de zapatos.

El poliuretano es un material sólido que puede ser poroso dependiendo de la densidad del mismo, éstos se obtienen mediante una reacción exotérmica que ocurre entre un poliol y un isocianato principalmente (Pervez y cols. 2018).

Una empresa productora de poliuretano ubicado en el municipio de Jilotepec (Estado de México), presento la necesidad de una mejora continua en sus productos en cuanto a calidad se refiere, ya que generalmente surgen problemas en la elaboración de algunos códigos de espuma de poliuretano como son el caso de la espuma 24 RFN y $24 \mathrm{FV}$, las cuales deben cumplir con densidad y compresión específicas. Por lo que resulta muy importante establecer una formulación que cumpla con los requisitos especificados, en este caso el poliuretano de interés está dirigido para la industria automotriz.

Aunque pueden existir diferentes formulaciones de dichas espumas, es importante encontrar la que mejor se adapte tanto a las necesidades de la empresa como las del cliente.

Por lo que es necesario explorar e identificar los factores significantes para la densidad y compresión ya que a partir de esto puede ser posible hallar la mejor formulación.

El presente proyecto se centra principalmente en el diseño de experimentos estadísticos, que ayude a la mejora de la espuma de poliuretano de los códigos de espuma $24 \mathrm{FV}$ y 24 RFN, se pretende lograr aplicando el método Taguchi, el cual es un método basado en un diseño experimental de arreglo ortogonal que utiliza información existente del proceso y que se realiza en una serie sistemática de etapas. (Sukthomya \& Tannock, 2005). Además de www.ecorfan.org/taiwan definir la mejor formulación que cumpla con la densidad y compresión necesarias.

\section{Espuma de poliuretano}

Es un material sintético y duroplástico, altamente reticulado y no fusible que se forma básicamente por la reacción química de dos compuestos, un poliol y un isocianato, aunque su formulación necesita y admite múltiples variantes y aditivos. Dicha reacción libera unos gases, (dióxido de carbono) que son los que van formando las burbujas. (Al, 2005). Se pueden clasificar tres tipos de espumas de poliuretano según su densidad: espumas flexibles, espumas rígidas y elastómeros.

\begin{tabular}{|l|l|l|}
\hline \multicolumn{1}{|c|}{ Espuma } & \multicolumn{1}{c|}{ Características } & \multicolumn{1}{c|}{ Usos } \\
\hline $\begin{array}{l}\text { Espumas } \\
\text { flexibles }\end{array}$ & $\begin{array}{l}\text { Las densidades } \\
\text { varían en un } \\
\text { intervalo de } 10 \text { a } \\
80 \mathrm{Kg} / \mathrm{m}^{3}\end{array}$ & $\begin{array}{l}\text { Fabricación de sillas, } \\
\text { muebles y colchones. }\end{array}$ \\
\hline $\begin{array}{l}\text { Espumas } \\
\text { rígidas }\end{array}$ & $\begin{array}{l}\text { Presentan } \\
\text { densidades entre } \\
30 \text { y } 50 \mathrm{Kg} / \mathrm{m}^{3}\end{array}$ & Aislante térmico \\
\hline Elastómeros & $\begin{array}{l}\text { Densidad, } \\
\text { usualmente } \\
\text { superiores a } 100 \\
\text { Kg/m }\end{array}$ & $\begin{array}{l}\text { Partes de vehículos o } \\
\text { para la fabricación de } \\
\text { suelas de zapatos }\end{array}$ \\
& & \\
\hline
\end{tabular}

Tabla 1 Tipos de espuma

\section{Materias primas}

El poliuretano flexible está fabricado esencialmente de los siguientes productos: Poliol (Polioxipropilenglicol) en un 55\% a $70 \%$ aproximadamente, TDI (Di-isocianato de Tolueno) en un $25 \%$ a $35 \%$, Agua, Catalizador metálico (Octoato Estañoso), Catalizador amínico, Surfactante o estabilizador, Agente soplante auxiliar, Colorantes y Aditivos (Retardantes a la flama, antiestáticos, antioxidantes, etc.).

Uno de los métodos más utilizados para la elaboración de las espumas de poliuretano es el denominado "one-shot", el cual corresponde al mezclado directo de los reactivos y demás componentes de la formulación, incluyendo catalizadores, estabilizadores, agentes de espumado y diversos aditivos. Las cantidades de cada componente dependen de una relación estequiométrica, en la que se calculan los equivalentes químicos necesarios para que la reacción de condensación se lleve a cabo con una eficiencia del 100\%, y también se debe considerar la cantidad de agua que se añadirá para promover la formación de dióxido de 
carbono $\left(\mathrm{CO}_{2}\right)$, el cual se aprovecha como agente espumante.

\section{Metodología Taguchi}

Taguchi establece que la calidad de un producto debe ser medida en términos de abatir al mínimo las pérdidas que ese producto le trae a la sociedad, desde que inicia su fabricación hasta que concluye su ciclo de vida; estas pérdidas sociales se traducen en pérdidas de la empresa en el mediano y largo plazos (Nasir y cols. 2013).

El objetivo del diseño robusto de parámetros es lograr productos y procesos ro bustos frente a las causas de la variabilidad (ruidos), que hacen que las características funcionales de los productos se desvíen de sus valores óptimos provocando costos de calidad (Rahul y Pretesh, 2018).

El concepto parámetro se refiere a los parámetros del sistema, es decir, son los factores o variables del proceso. Decimos que un producto o proceso es robusto cuando su funcionamiento es consistente al exponerse a las condiciones cambiantes del medio. (Altan, 2010).

Existen 3 etapas para el desarrollo de un producto: Diseño del sistema, Diseño de parámetros, Diseño de tolerancias

\section{Metodología a desarrollar}

\section{Procedimiento general para la elaboración de espuma de poliuretano}

Las mezclas de los ingredientes se realizaron en recipientes de plástico de $5 \mathrm{~L}$, en los cuales se pesaron inicialmente los polioles según lo establecido en la formulación, se vertió la cantidad en gramos de agua, silicón, amina y color según lo indicado. Posteriormente se mezclaron durante 90 segundos, una vez realizada esta primera mezcla se agregó el catalizador y se volvió a mezclar.

Para la mezcla de los componentes se utilizó taladro Bosch de velocidad variable de 0 a 3000rpm, con un agitador de hélice cubierta.

Por último, se pesó y agrego a la mezcla el TDI según la formulación y se mezcló durante 6 segundos, (se comenzó a tomar el tiempo a partir de este momento como inicio de la reacción); para finalmente ser vaciado en un cajón de madera forrado con plástico (molde final en forma de prisma rectangular).

Después de verter el material, se observó el momento en el cual el producto empezó a expandir, instante que se registró como "tiempo de crema", hasta que alcanzó el punto máximo, cuando se observó el momento en que la espuma libero $\mathrm{CO}_{2}$ y se registró como tiempo final de reacción.

Concluido este instante, se etiquetaron y se dejaron secar las muestras durante un día para luego registrar su altura y ser cortadas para obtener cuadros de $34 \times 34 \times 10 \mathrm{~cm}$, para poder evaluar la densidad y compresión de las muestras.

Para el cálculo de la densidad, se pesaron cada una de las muestras obtenidas en una báscula analítica y se obtuvo el volumen de cada una de ellas. Se halló la densidad con la siguiente fórmula:

$$
\frac{\text { Peso.Espuma. }(\mathrm{g})}{\operatorname{LxA} \mathrm{E} \cdot\left(\mathrm{cm}^{3}\right)} \times \frac{1 \mathrm{Kg}}{1000 \mathrm{~g}} \times \frac{1,000,000 \mathrm{~cm}^{3}}{1 \mathrm{~m}^{3}}=\text { Densidad }\left(\frac{\mathrm{Kg}}{\mathrm{m}^{3}}\right)
$$

Finalmente, se evaluo la identación de las muestras utilizando una maquina universal marca Instron®. Cabe mencionar que la identación se define como la fuerza necesaria para comprimir un área determinada de espuma de poliuretano. Específicamente los valores más utilizados son $25 \%$ ID, el cual es la fuerza necesaria para comprimir la espuma al $25 \%$ de su espesor o altura original, y $65 \%$ ID, que de igual manera es la fuerza necesaria para comprimir la espuma al $65 \%$ de su espesor o altura original. (Al, 2005; Nasir y cols., 2013).

Durante la fase inicial se utilizó una formulación base para los ensayos en la cual se realizaron las modificaciones en las materias primas. Luego de verificar el comportamiento e influencia de las mismas en las propiedades finales de la espuma, se utilizó este conocimiento para proponer una formulación para una empresa del sector de los poliuretanos con condiciones específicas de desempeño.

\section{Primer arreglo ortogonal para los Códigos de espuma 25 STD BCQ y 64 FB}

El diseño experimental se llevó a cabo mediante el uso del software estadístico Minitab® v.16 con el cual se desarrollaron los arreglos ortogonales L8 (con 1 réplica) para la 
formulación de las espumas de poliuretano (Vinitkumar, 2017).

Una vez que se establecieron los factores fue necesario delimitar los niveles, tanto mínimo como máximo, de cada parámetro involucrado en cada una de las pruebas y así poder generar la formulación necesaria para realizar las pruebas de cajón de $2 \mathrm{~kg}$ a nivel laboratorio.

\begin{tabular}{|c|c|c|c|c|c|c|}
\hline No & $\begin{array}{l}\text { Poliol } \\
\text { Graf }\end{array}$ & TDI & Agua & Silicón & Amina & Catalizador \\
\hline 1 & 70 & 1.16 & 2.8 & 0.8 & 0.07 & 0.15 \\
\hline 2 & 70 & 1.16 & 2.8 & 1 & 0.09 & 0.17 \\
\hline 3 & 70 & 1.18 & 3 & 0.8 & 0.07 & 0.17 \\
\hline 4 & 70 & 1.18 & 3 & 1 & 0.09 & 0.15 \\
\hline 5 & 75 & 1.16 & 3 & 0.8 & 0.09 & 0.15 \\
\hline 6 & 75 & 1.16 & 3 & 1 & 0.07 & 0.17 \\
\hline 7 & 75 & 1.18 & 2.8 & 0.8 & 0.09 & 0.17 \\
\hline 8 & 75 & 1.18 & 2.8 & 1 & 0.07 & 0.15 \\
\hline
\end{tabular}

Tabla 2 Arreglo ortogonal L8 con réplica de la espuma 25 STD BCQ

\begin{tabular}{|r|r|r|r|r|r|r|r|}
\hline No & $\begin{array}{l}\text { Poliol } \\
\text { Graf }\end{array}$ & \multicolumn{2}{c|}{ TDI } & \multicolumn{2}{|c|}{$\begin{array}{r}\text { Agua } \\
\text { Setar- } \\
\text { dante } \\
\text { Flama }\end{array}$} \\
\hline 1 & 18 & 1.14 & 3 & 1.1 & 0.11 & 0.12 & 9 \\
\hline 2 & 18 & 1.14 & 3 & 1.3 & 0.13 & 0.14 & 11 \\
\hline 3 & 18 & 1.16 & 3.3 & 1.1 & 0.11 & 0.14 & 11 \\
\hline 4 & 18 & 1.16 & 3.3 & 1.3 & 0.13 & 0.12 & 9 \\
\hline 5 & 22 & 1.14 & 3.3 & 1.1 & 0.13 & 0.12 & 11 \\
\hline 6 & 22 & 1.14 & 3.3 & 1.3 & 0.11 & 0.14 & 9 \\
\hline 7 & 22 & 1.16 & 3 & 1.1 & 0.13 & 0.14 & 9 \\
\hline 8 & 22 & 1.16 & 3 & 1.3 & 0.11 & 0.12 & 11 \\
\hline
\end{tabular}

Tabla 3 Arreglo ortogonal L8 con réplica de la espuma 64 FB

\section{Resultados}

\section{Análisis estadístico para código 25 STD BCQ densidad e identación.}

Una vez realizadas las muestras de espuma de poliuretano fue necesario evaluar propiedades como la densidad e identación para poder determinar los factores significantes. realizar el análisis de varianza (tanto para densidad como para identación) así como los gráficos de efectos principales $(\alpha=0.05)$.

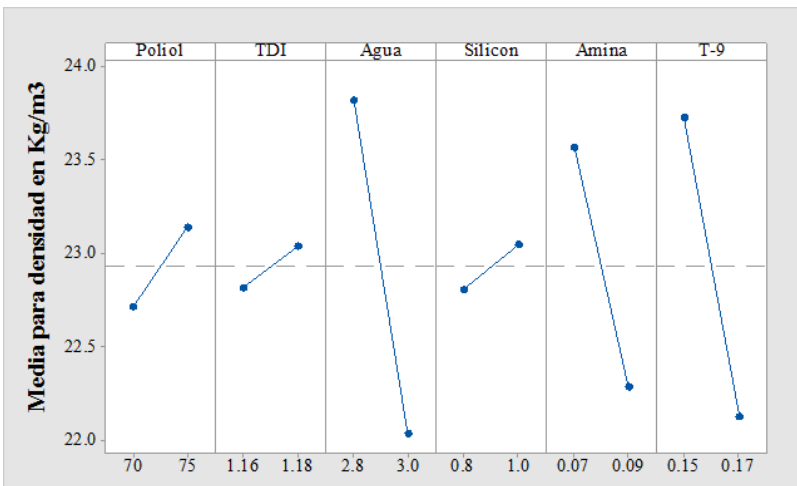

Gráfico 1 Gráfica de efectos principales para medias en la densidad en la espuma 25 STD BCQ

Como se puede observar en la Gráfica 1, los factores: agua, amina y catalizador presentaron mayor significancia descriptiva sobre la cualidad de densidad de la espuma 25 STD BCQ.

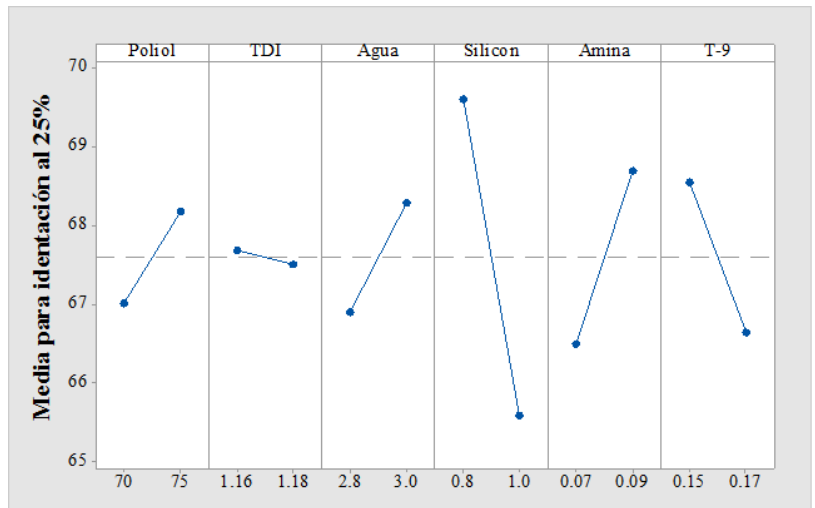

Gráfico 2 Gráfica de efectos principales para medias en identación en la espuma 25 STD BCQ

Se puede observar que en la Gráfica 2, que los factores más significativos en la propiedad de identación son: agua, amina y silicón para la espuma 25 STD BCQ. Derivado de lo anterior se procedió a generar un nuevo diseño ortogonal L9 con los siguientes factores, con niveles en ppm:

\begin{tabular}{|l|r|r|r|}
\hline \multicolumn{1}{|c}{ Factor } & \multicolumn{1}{c}{ Nivel 1 } & \multicolumn{1}{c}{ Nivel 2 } & \multicolumn{1}{c|}{ Nivel 3 } \\
\hline Amina & 0.07 & 0.08 & 0.09 \\
\hline Silicón & 0.8 & 0.9 & 1.0 \\
\hline
\end{tabular}

Tabla 4 Arreglo ortogonal L8 con réplica de la espuma 25 STD BCQ

Luego de realizadas las pruebas del segundo arreglo ortogonal, se realizó un análisis con gráfica de contorno (Gráfica 3) en este caso, dicha grafica hace referencia a la densidad conforme a la variación de la amina y al silicón, en la cual se puede observar un panorama de predicción para la densidad que queremos implementar en la espuma 25 STD BCQ; cómo se puede observar se puede elegir un nivel de 0.080 , 0.084 y 0.088 ppm de amina y un nivel de 0.90 a 0.95 ppm para el silicón debido a que son los que se encuentran en la zona clara del centro ya que según la gráfica de contorno son los valores con los que se puede obtener la densidad menor o igual de $24 \mathrm{~kg} / \mathrm{m}^{3}$. 


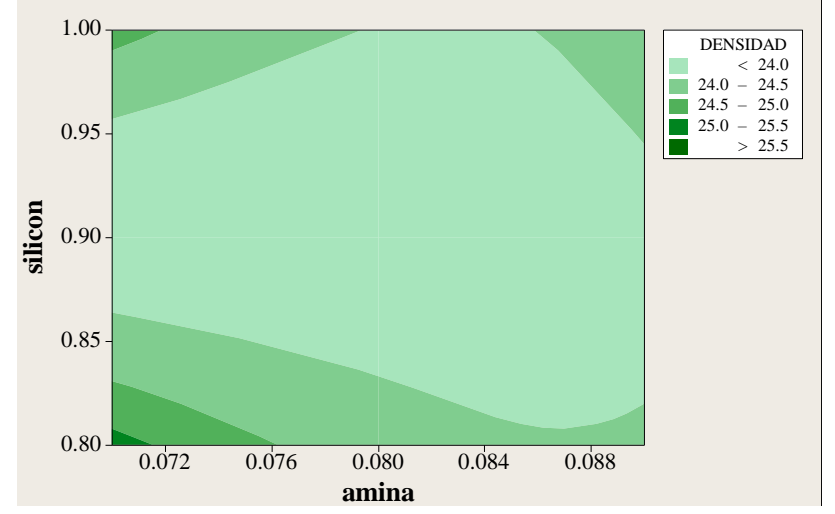

Gráfico 3 Gráfica de contornos para la densidad en la espuma 25 STD BCQ

Para poder elegir el valor correcto es necesario realizar el análisis con la gráfica de contorno para identación para poder elegir el nivel que más con convenga.

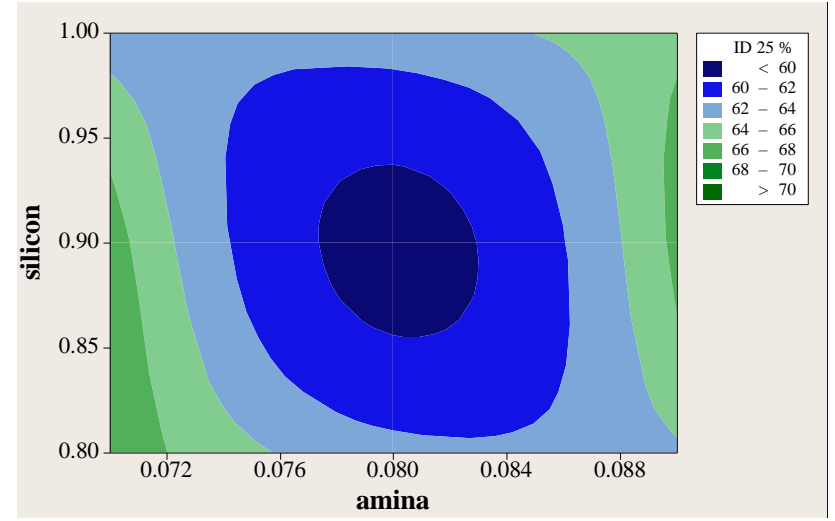

Gráfica 4 Gráfica de contornos para identación en la espuma 25 STD BCQ

Como se mencionó anteriormente, en el análisis llevado a cabo de la espuma 25 STD $\mathrm{BCQ}$, para el segundo arreglo ortogonal se modificaron dos factores que fueron la amina y el silicón y por ende resultaron dos graficas de contorno. En la gráfica de contorno para la identación (Gráfica 4) se puede observar el panorama de predicción para la identación según se varié la amina y el silicón; con ayuda de esta grafica se pudo elegir un nivel de 0.08 en la amina y de 0.9 en el silicón (al igual que para la densidad) para así asegurar que fijando estos niveles se obtendría una identación entre 57 y 63 lbf, el cual es un parámetro optimo en la calidad de la espuma 25 STD BCQ.

\section{Análisis estadístico para código 64 FB densidad e identación}

Una vez realizadas las muestras de espuma de poliuretano fue necesario evaluar propiedades como la densidad e identación para poder determinar los factores significantes.

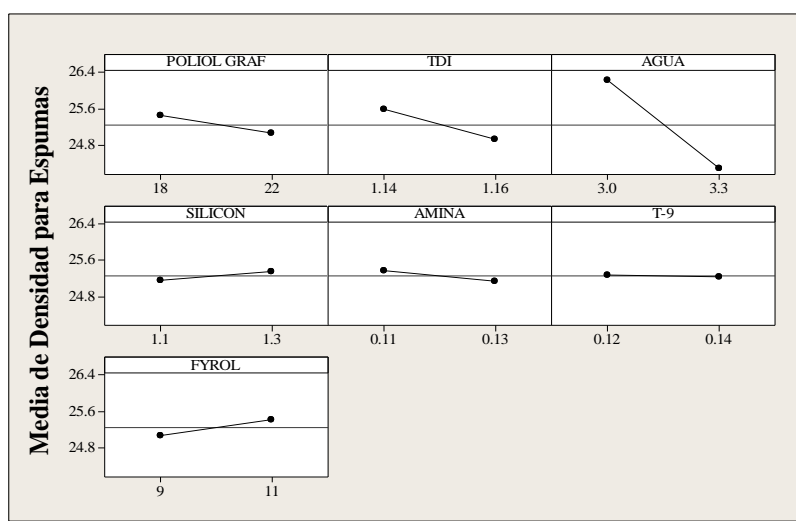

Gráfica 5 Gráfica de efectos principales para medias en la densidad en la espuma 64 FB

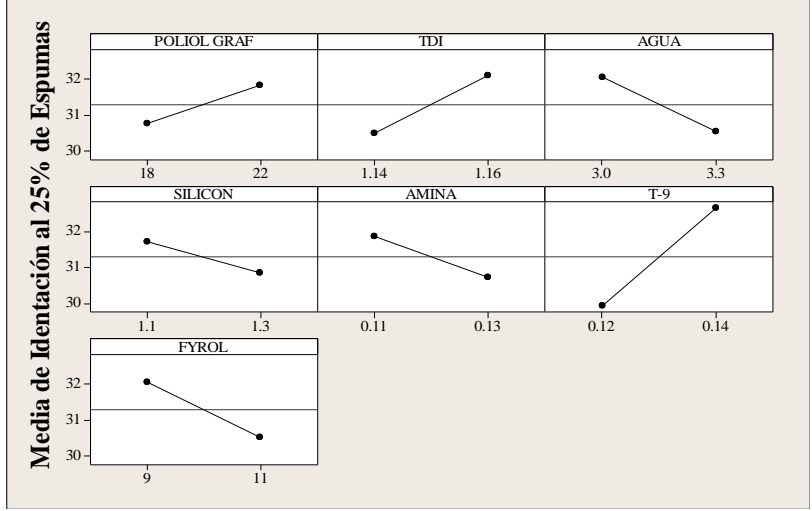

Gráfica 6 Gráfica de efectos principales para medias en la densidad en la espuma 64 FB

Para la propiedad de densidad, se hace la clasificación de factores de mayor a menor de acuerdo a la importancia que tienen respecto a la densidad, como es de esperarse el agua sigue siendo el principal factor seguido del índice de TDI y el poliol. Para la propiedad de identación al 25\%. al análisis de medias realizado para la identación se puede observar que los factores más significantes son: catalizador, índice de TDI y poliol (Graficas 5 y 6).

Analizado el criterio anterior se prosiguió a realizar el segundo arreglo ortogonal dejando fijos los niveles de agua, amina $\mathrm{y}$ catalizador y modificando el resto de los factores, pero ahora se tomarían en cuenta 3 niveles para cada factor (índice de TDI, poliol graf, retardante de flama y silicón) lo que nos daría un arreglo L9 (con réplica) como se muestra a continuación (niveles en ppm):

\begin{tabular}{|l|r|r|r|}
\hline \multicolumn{1}{|c|}{ Factor } & \multicolumn{1}{c}{ Nivel 1 } & \multicolumn{1}{c|}{ Nivel 2 } & Nivel 3 \\
\hline Índice TDI & 1.14 & 1.15 & 1.16 \\
\hline Poliol & 18 & 20 & 22 \\
\hline Retardante de flama & 9 & 10 & 11 \\
\hline Silicon & 1.1 & 1.2 & 1.3 \\
\hline
\end{tabular}

Tabla 5 Arreglo ortogonal L8 con réplica de la espuma 64 FB

LAGUNAS-BERNABÉ, Ignacio, HUITRÓN-CID, María De Lourdes, ALVARADO-ORTIZ, Jesús y PÉREZ-MIRELES, Emilio. Optimización del proceso industrial del poliuretano a través del diseño estadístico de Taguchi. Revista de Tecnologías en Procesos Industriales. 2019 
Obtenidos los resultados de la densidad y la identación se hizo el correspondiente análisis estadístico (superficie de respuesta), obteniéndose las siguientes graficas de contorno, en las cuales se mostrarían los niveles óptimos para predecir la identación y la densidad deseada.

Como se puede observar en esta gráfica 7 , se desea alcanzar una densidad promedio entre 23.6 y $24 \mathrm{~kg} / \mathrm{m}^{3}$ por lo que es necesario ajustar los niveles de índice de TDI entre los valores de 1.14 a 1.1415 aproximadamente y de POLIOL GRAF entre los valores de 18 a 22.

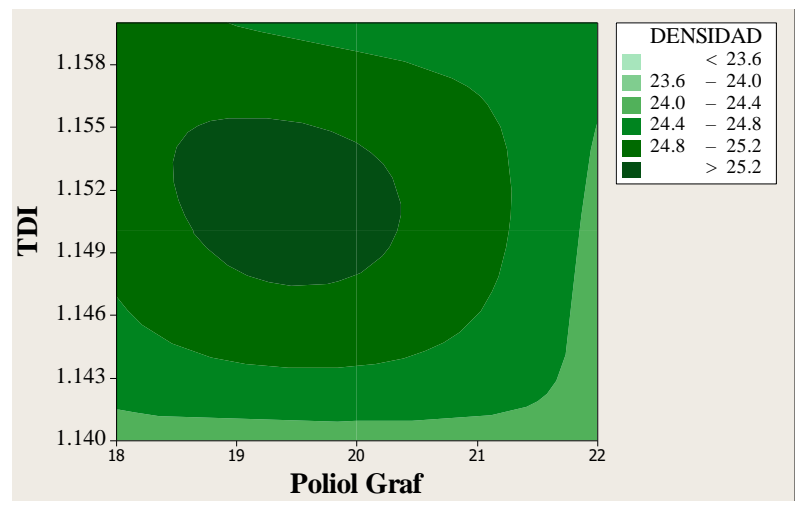

Gráfica 7 Gráfica de contornos para densidad en la espuma 64 FB

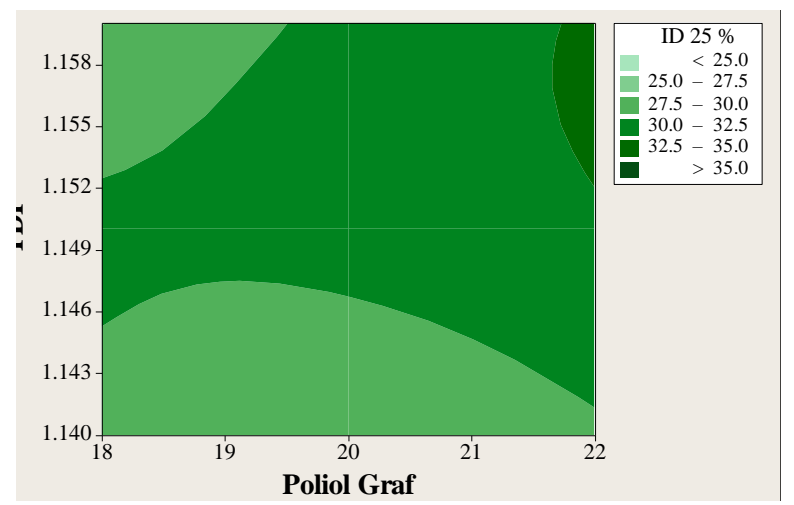

Gráfica 8 Gráfica de contornos para identación en la espuma 64 FB

Para la gráfica 8, se analiza ahora como varia la identación conforme los niveles de TDI y Poliol Graf, en dicha grafica se muestra el área de interés con gris para un identación entre 25 y 27.5 lbf, hallándose que el TDI debe mantenerse entre 1.14 a 1.145 y de poliol Graf entre 18 y 22 respectivamente para obtener dicha identación.

\section{Conclusiones}

Se observó que la densidad en ambos códigos de espuma es afectada por la cantidad de agua que contiene cada formulación.
Como se ha mencionado antes, el agua juega un papel fundamental al ser un agente soplante, éste reacciona con el isocianato para producir $\mathrm{CO}_{2}$ que apertura las celdas de la espuma permitiendo un mayor tamaño, por lo tanto, es posible decir que a mayor cantidad de agua menor densidad y viceversa (Shuaib y cols., 2011).

En cuanto al factor principal que interviene en la identación de la espuma 25 STD BCQ es el silicón, cuya función es ofrecer una menor tensión superficial y por lo tanto ayudan a aumentar el número de burbujas de aire introducidas durante la mezcla; estas burbujas de aire actúan como punto de partida para el crecimiento de las celdas generándolas de un menor tamaño (Ahmad y cols., 2016). A diferencia de esta espuma, el código 64 FB se vio afectada por el catalizador, que de acuerdo con lo observado dentro de la empresa además de acelerar la reacción, también afecta en la apertura de las celdas de la espuma haciéndolas más duras y agrietadas en ocasiones (Gurjeet y cols., 2015).

Para ambos códigos fue necesario realizar un segundo arreglo ortogonal para poder fijar los niveles óptimos para las formulaciones finales. En la formulación 25 STD BCQ se logró establecer que el nivel de catalizador, agua, índice de TDI y Poliol se podían mantener fijos sin afectar ninguna de las dos propiedades evaluadas, y así solo se reajustarían los niveles de los factores de silicón y amina, logrando estandarizar un nivel óptimo mediante las gráficas de contorno estableciendo un novel de 0.08 ppm de amina y 0.9 ppm de silicón y con ello se logró llegar a una mejor formulación, la cual aseguraría una densidad de $23.96 \mathrm{Kg} / \mathrm{m}^{3}$ y una identación al $25 \%$ de 63.14 lbf, los cuales son parámetros dentro de la normatividad de esta espuma.

\section{Referencias}

Ahmad, N.; Kamal, S.; Raza, Z.A.; Hussain, T.; Anwar, F. (2016). Multi-response optimization in the development of oleo-hydrophobic cotton fabric using Taguchi based grey relational analysis. Applied Surface Science. 367:370-381.

Al., L. J. (2005). Thermal Stability and Fire Properties of Conventional Flexible Polyurethane Foam Formulations. Polymer Degradation and Stability, 88: 256-261.

LAGUNAS-BERNABÉ, Ignacio, HUITRÓN-CID, María De Lourdes, ALVARADO-ORTIZ, Jesús y PÉREZ-MIRELES, Emilio. Optimización del proceso industrial del poliuretano a través del diseño estadístico de Taguchi. Revista de Tecnologías en Procesos Industriales. 2019 
Altan M. (2010). Reducing shrinkage in injection moldings via Taguchi, Anova and neural network methods., Materials and design;31;599-604

Gurjeet Singh, M. K. Pradhan, Ajay Verma. (2015). Effect of Injection Moulding Process Parameter on Tensile Strength Using Taguchi Method. International Journal of Mechanical, Aerospace, Industrial, Mechatronic and Manufacturing Engineering Vol:9, No:10.

Kamaruddin S., Zahid A., Foong S.H. (2010). Application of Taguchi Method in the optimization of injection moulding parameters for manufacturing products from plastic blend. IACSIT, 2(6): 574-580

Nasir S.M., Ismail K.A., Shayfull Z. Derus M.A. (2013). Warpage improvement of thick component using Taguchi optimization method for single and double gates in injection molding process. Australian Journal of Basic and Applied Sciences, 7(5): 205-212.

Pervez M.N., Shafiq F., Sarwar Z., Jilani M.M., Cai Y. (2018). Multi-response optimization of resin finishing by using a Taguchi-based grey relational analysis. Materials. 11(426):1-19

Rahul D. y Pretesh J. (2018). Application of Taguchi-based design of experiments for industrial chemical process. En Statistical Approaches with emphasis on design of experiments applied to chemical processes. INTECH, pp 1-21.

Shuaib, N. A., M. F. Ghazali, Z. Shayfull, M.Z. M. Zain, and S. M. Nasir. (2011). Warpage Factors Effectiveness of a Thin Shallow Injection-Molded Part Using Taguchi Method. International Journal of Engineering and Technology 11(1) : 182-87.

Vinitkumar K.M. (2017). Analysis of use of Taguchi method with design of experiment (DOE) and analysis of variance (ANOVA) in plastic injection molding for quality improvement by optimization. IJRASET 5(7):136-144 